\title{
Medium-term Rail Planning at Rio Tinto Iron Ore
}

\author{
R. García-Flores ${ }^{a}$, G. Singh ${ }^{\mathrm{a}}$, A. Ernst ${ }^{\mathrm{a}}$ and P. Welgama ${ }^{\mathrm{b}}$ \\ ${ }^{a}$ CSIRO Mathematics, Informatics and Statistics, Private Bag 33, Clayton South, VIC, \\ Australia, 3169 \\ ${ }^{\mathrm{b}}$ Rio Tinto Technical Services, GPO Box A42, Perth, WA, Australia, 6837 \\ Email: Rodolfo.Garcia-Flores@csiro.au
}

\begin{abstract}
In minerals supply chains, medium term plans over time horizons from two weeks to two years are used not only to maximise throughput and identify bottlenecks, but also for scheduling crews, production and maintenance. These plans need to observe constraints like maintenance and production requirements, fleet capacities, and dumping, loading and stockyard management at every site. Additionally, the iron ore supply chains considered in this paper also needs to deal with grade quality constraints. This introduces extra complexities in terms of non-linear constraints as the quality depends on the mixing ratio of ore from different sources. We present an optimisation tool that was developed for Rio Tinto Iron Ore for such planning. The tool provides optimal number of trains while taking into account all the system constraints and has already reduced total planning time significantly.
\end{abstract}

Keywords: Scheduling, Rail planning, Medium-term planning, Blending, Mixed-integer nonlinear programming 


\section{INTRODUCTION}

Australia is the largest iron ore exporter in the world. In 2009 it produced 394 million tonnes of iron ore, of which 362 million tonnes were exported, generating $\$ 30$ billion in value. Of this material, 97\% came from the Pilbara region of Western Australia (WA). Rio Tinto Iron Ore (RTIO) is one of the major producers, with an operating capacity of around 240 million tonnes per annum. Its freight rail network, the largest privately-owned in Australia, currently consists of twelve mines and three shipping terminals, with plans to further expand. Planning operations for such an extensive network has proved to be a cumbersome and lengthy process that needs manual estimations of weekly trains, and where preservation of iron ore grade quality is a nontrivial requirement. An additional disadvantage of the manual approach is the rigidity of the results, which make it very difficult to undertake a "what-if" analysis. CSIRO developed a model that finds the optimal production schedule, assists in medium-term planning of operations, and enables the assessment of alternative scenarios. It was implemented in $\mathrm{C}++$ with the capability of interfacing to Gurobi or CPLEX with a Visual Basic-supported user interface in Excel.

Several medium-term planning projects have been reported in the literature. Li and Tayur 2005 present a rail scheduling problem that incorporates pricing into a medium-term planning of intermodal transportation. Their focus is on pricing, in contrast with our emphasis on grade compliance. Bilgen and Ozkarahan 2007] adopt a very similar modelling approach for determining the amount of blended grain shipped between ports, as well as inventory bookkeeping. Data sparsity (coming for example from unconnected ports) is exploited by compacting data sets and querying SQL selectively. For our project, non-relevant combinations of set elements, as for example car dumpers working at one but not other ports, are filtered as part of the design of the $\mathrm{C}++$ program. A crucial difference is that all mixtures in Bilgen and Ozkarahan 2007] are binary, whereas in our case product blends involve ten components.

Projects involving blending and planning usually deal with these problems separately. For example, Sandeman and Stanford [2010] discuss a mineral export optimisation model coupled to a discrete-event simulation module used to fine-tune the grades of the mineral. Rehman and Asad 2010 discuss an optimal quarry production scheduling problem. Quarry block extraction requirements are modelled as precedence constraints and savings are compared to an empirical approach. Ulstein et al. 2007] formulate a model for the Norwegian oil industry where the mole fraction of different hydrocarbons is considered while optimising the oil and gas distribution plans. Their model improves the way flow from the wells is processed and distributed. The study of Fishman and McInnes 2005] describes a coal supply chain analysis tool that emphasises sampling and quality control for coal operations.

Our medium-term rail planning model is introduced formally in Section 2, emphasising the calculations needed to preserve grade quality. Section 3 describes the optimal and "roll-over" heuristic approaches to the solution and their relative performance. Section 4 presents the results and Section 5 rounds the discussion with conclusions and future work.

\section{PLANNING MODEL}

In many mining export problems, buffers (stockpiles) serve to manage variabilities in product quality and in the amount of transported materials. Stockpiles in mines and ports are used for blending the ore into products that meet the quality specifications, as well as to control the flow of material through the system. Products mined at various sites can be roughly classified as lump and fines, each with its own particular composition. Subsection 2.3 will present a more detailed description of how blending is handled as part of the planning process.

\subsection{Objective Function}

The objective is to maximise revenue while minimising the deviation from the product quality specification, 
Revenue $=$ Total profit - Cost of live stockpile violations - Cost of bulk stockpile violations

- Cost of grade non-compliance - Cost of bulk handling

- Cost of extra-trains - Cost of violating preference requirements

- Cost of violating return fines requirements.

The total profit for shipping product is $\sum_{s \in S} S P_{s} \sum_{t \in T} \sum_{r \in R} z_{r, s, t}$, where $S P_{s}$ is the profit per shipped tonne of product $s$ and $z_{r, s, t}$ is the amount of product $s$ shipped from port $r$ in period $t$. The cost of live stockpile limit violations is

$$
\sum_{m \in M} \sum_{p \in P_{m}} M S V L_{m, p} \sum_{t \in T}\left(\alpha_{m, p, t}^{\uparrow}+\alpha_{m, p, t}^{\downarrow}\right)+\sum_{r \in R} \sum_{s \in S_{r}} P S V L_{r, s} \sum_{t \in T}\left(\beta_{r, s, t}^{\uparrow}+\beta_{r, s, t}^{\downarrow}\right),
$$

where $M S V L_{m, p}$ represents the limit violation penalty for live stockpiles of mined product $p$ in mine $m, P S V L_{r, s}$ is the limit violation penalty for live stockpiles in the ports, and $\alpha_{m, p, t}^{\uparrow}$ and $\alpha_{m, p, t}^{\downarrow}$ are the amounts by which the maximum and minimum live stockpile limits were violated, respectively. An analogous term is needed for the cost of bulk stockpile limit violations.

The cost of deviating from the target compositions of the shipped products is

$$
\sum_{c \in C} \sum_{r \in R} \sum_{s \in S_{r}}\left[G P I_{r, s, c} \sum_{t \in T}\left(s i_{r, s, c, t}+e i_{r, s, c, t}\right)+G P O_{r, s, c} \sum_{t \in T}\left(s o_{r, s, c, t}+e o_{r, s, c, t}\right)\right],
$$

where $G P I_{r, s, c}$ is the penalty for violating the target grade of component $c$ and $G P O_{r, s, c}$ is the penalty for violating the composition control limit, $s i_{r, s, c, t}$ and $e i_{r, s, c, t}$ are the slack and excess variables to penalise when $c$ is off target, respectively, and $s i_{r, s, c, t}$ and $e i_{r, s, c, t}$ are the slack and excess variables to penalise when $c$ is outside the control limit, respectively. The cost associated to moving material from/to bulk stockpiles is

$$
\sum_{m \in M} \sum_{p \in P_{m}} B P M_{m, p} \sum_{t \in T}\left(y_{m, p, t}^{+}+y_{m, p, t}^{-}\right)+\sum_{r \in R} \sum_{s \in S_{r}} B P R_{s, r} \sum_{t \in T}\left(u_{r, s, t}^{+}+u_{r, s, t}^{-}\right),
$$

where $B P M_{m, p}$ and $B P R_{r, s}$ are the handling costs of products at mines and ports respectively, $y_{m, p, t}^{+}$and $y_{m, p, t}^{-}$are the transfers to and from bulk stockpiles at mines, and $u_{r, s, t}^{+}$and $u_{r, s, t}^{-}$are the transfers to and from bulk stockpiles at ports.

The cost of exceeding train trips is $\sum_{f \in F} T P_{f} \sum_{t \in T} \gamma_{f, t}$, where $\gamma_{f, t}$ is the amount by which the number of allowed trains of fleet $f$ was exceeded, and $T P_{f}$ is the corresponding penalty.

The cost of not respecting a preference like dumping a product in a specific car dumper is expressed as $\sum_{m \in M} \sum_{d \in D_{m}} P P_{m, d} \sum_{t \in T} \kappa_{m, d, t}$, where $P P_{m, d}$ denotes the penalty for not fulfilling the preference and $\kappa_{m, d, t}$ is the amount by which a selected car dumper $d$ in port $r$ could not comply with dumping all product $p$.

Finally, the penalties for violating the preferred ratio of shipped lump to fines (see subsection 2.3) are $\sum_{r \in R} P R S P_{r} \sum_{t \in T} \delta_{r, t}$, where $P R S P_{r, t}$ is the penalty for violating the specified ratio, and $\delta_{r, t}$ is the penalty variable for deviations in the ratio.

\subsection{Operational constraints}

The main constraints that must be followed so that the schedule is operationally feasible are:

1. The total amount of live product stacked, both at the mines and the terminal, should not exceed the allocated product capacities and the live stockpile capacity. Live stockpiles are part of the main production line and bulk stockpiles act as buffers.

$$
\begin{aligned}
S_{m, p, t}^{\min }-\alpha_{m, p, t}^{\downarrow} \leq & s_{m, p, t} \leq S_{m, p, t}^{\max }+\alpha_{m, p, t}^{\uparrow} \quad \forall m \in M, p \in P_{m}, t \in T \\
W_{r, s, t}^{\min }-\beta_{r, s, t}^{\downarrow} \leq w_{r, s, t} & \leq W_{r, s, t}^{\max }+\beta_{r, s, t}^{\uparrow} \quad \forall r \in R, s \in S_{r}, t \in T,
\end{aligned}
$$


where $s_{m, p, t}$ and $w_{r, s, t}$ are the live stockpile levels at mines and ports respectively, $S_{m, p}^{\min }$ and $S_{m, p}^{\max }$ are the minimum and maximum live stockpile levels at mine $m$, and $W_{r, s}^{\min }$ and $W_{r, s}^{\max }$ are the minimum and maximum live stockpile levels at port $r$. Similar constraints apply to bulk product.

2. Inloaders and outloaders cannot service more than a specified maximum capacity in tonnes per period.

$$
\begin{array}{lcll}
0 \leq & \sum_{s \in S} z_{r, s, t} & \leq Z_{r, t}^{\max } & \forall r \in R, s \in S_{r}, t \in T \\
0 \leq & s_{m, p, t} & \leq Y_{m, p}^{\max } & \forall m \in M, p \in P_{m}, t \in T \\
0 \leq & w_{r, s, t} & \leq U_{r, s}^{\max } & \forall r \in R, s \in S_{r}, t \in T,
\end{array}
$$

where $Z_{r, t}^{\max }, U_{r, s}^{\max }$ and $Y_{m, p}^{\max }$ are the maximum tonnes of product that can be shipped, stockpiled in ports and stockpiled in mines, respectively.

3. The live stockpiles must not exceed the site's yard limit. For mines and ports that have a bulk stockpile, material accumulates in the live stockpile until reaching its maximum control limit, then it is placed into the bulk stockpile until this reaches its capacity, and then it is put again in the live stockpile until this reaches the stockyard limit. For mines,

$$
\begin{aligned}
S_{m, p, t}^{\max }+\alpha_{m, p, t}^{\uparrow} & \leq Y L M_{m, p, t} \quad \forall m \in M, p \in P_{m}, t \in T \\
y_{m, p, t}^{+} & \geq \alpha_{m, p, t}^{\uparrow} \quad \forall m \in M, p \in P_{m}, t \in T,
\end{aligned}
$$

where $Y L M_{m, p, t}$ is the yard limit. Similar constraints apply to ports.

4. The number of trains that service a region cannot exceed the total allowed number of trains in that region.

$$
\sum_{m \in g} \sum_{p \in P_{m}} \sum_{d \in D_{m, r, p}} \sum_{s \in S} x_{m, p, d, s, t} \leq M T_{g, t} \quad \forall g \in G, t \in T,
$$

where $x_{m, p, d, s, t}$ is the number of trains and $M T_{g, t}$ is the maximum number of allowed trains in region $g$. Similar capacity constraints apply to train fleets, car dumpers, inloaders at the mines and the ports and outloaders at the mines and at the ports.

5. The total number of consists and consist hours for each fleet should not exceed the available pooled fleets and hours of a fleet in a given period,

$$
\begin{aligned}
\sum_{m \in M_{f}} \sum_{p \in P_{m}} \sum_{d \in D_{m, r, p}} \sum_{s \in S} x_{m, p, d, s, t} & \leq M F T_{f, t}+\gamma_{f, t} \quad \forall f \in F, t \in T \\
\sum_{m \in M_{f}} \sum_{p \in P_{m}} C T_{m, p, t} \sum_{d \in D_{m, r, p}} \sum_{s \in S} x_{m, p, d, s, t} & \leq P F T_{f, t}+\mu_{f, t} \quad \forall f \in F, t \in T,
\end{aligned}
$$

where $C T_{g, p, t}$ is the cycle time of a train carrying mined product $p$ from region $g$ at period t, $M F T_{f, t}$ are the available consist numbers and $P F T_{f, t}$ is the available pooled hours of fleet $f$ at period $t$. The $\gamma_{f, t}$ are the additional consists required and $\mu_{f, t}$ is the additional cycle time needed for fleet $f$ at period $t$.

6. In the mines, the live stockpiles of product at the beginning of a period equal the existing material in the stockpile plus the produced material plus the material transferred from the corresponding bulk stockpile minus the material transferred to the corresponding bulk stockpile minus the material railed to the ports.

$$
\begin{aligned}
s_{m, p, t+1}=s_{m, p, t} & +I O P_{p, m, t}+y_{m, p, t}^{-}-y_{m, p, t}^{+} \\
& -T S_{m, p} \sum_{d \in D_{m, r, p}} \sum_{s \in S_{m, p}} x_{m, p, d, s, t} \quad \forall m \in M, p \in P_{m}, t \in T,
\end{aligned}
$$

where $T S_{m, p}$ is the capacity of a train in tonnes transporting product $p$ from mine $m$, and $I O P_{p, m, t}$ is the amount of product $p$ produced at mine $m$ in period $t$. Similar bookkeeping constraints apply to bulk at the mines and bulk and live material at the ports. 
Other constraints are concerned with special requirements, for example penalties for not delivering a specific product to a particular car dumper. These are not shown due to space limitations.

\subsection{Planning and blending}

Blending poses a special challenge. The model must comply not only with iron ore composition, but also with other requirements, like mixing a fraction of the fines with lump to enhance its quality. To illustrate only one of the non-linearities introduced by grades, we discuss railed material composition. Analogous expressions are needed for stockpiles at mines and ports.

Railed grade calculations. The model considers material handling regimes in the mines as one of first-in-last-out (FILO) and first-in-first-out (FIFO). If the mine is FILO then

$$
R G_{m, p, c, t}=\frac{\sum_{d \in D_{m, r, p}} \sum_{s \in S_{m, p}}\left(I O P \_t n s_{m, p, c, t}+L_{-} t n s_{m, p, c, t}+B \_t n s_{m, p, c, t}\right)}{\sum_{d \in D_{m, r, p}} \sum_{s \in S_{m, p}} T S_{m, p} x_{m, p, d, s, t-1}}
$$

where

$$
\begin{aligned}
I O P \_t n s_{m, p, c, t} & =\min \left\{T S_{m, p} x_{m, p, d, s, t-1}, I O P_{m, p, t-1}\right\} I O P \_g r a d e_{m, p, c, t-1} \\
L \_t n s_{m, p, c, t} & =\min \left\{s_{m, p, t-1}, \max \left\{0.0, T S_{m, p} x_{m, p, d, s, t-1}\right\}\right\} L M_{m, p, c, t-1} \\
B \_t n s_{m, p, c, t} & =\max \left\{0.0, T S_{m, p} x_{m, p, d, s, t-1}-I O P_{m, p, t-1}-s_{m, p, t-1}\right\} B M_{m, p, c, t-1} .
\end{aligned}
$$

The terms $I O P_{-}$grade $_{m, p, c, t-1}, L M_{m, p, c, t-1}$ and $B M_{m, p, c, t-1}$ represent the mined grade, live material grade, and bulk grade respectively. Equation (2) implies that, if the mine's regime is FILO, the produced quantity will first be loaded into the trains leaving the mine, and the remaining from the live stockpiles. If this amount is not sufficient, the remaining amount will come from the bulk stockpile. Accordingly, the respective grades are multiplied to accurately calculate the grade railed from the mine. An analogous expression is needed for mines whose regime is FIFO.

To obtain an approximate solution, an iterative method was used. In the first step, the problem was solved without grade constraints. In the following steps, the solution value for stockpile levels and number of trains of the previous iteration was fed back into the problem as estimates for the denominators for the calculation of the grades. This procedure was repeated for a fixed number of iterations.

\section{SOLUTION APPROACHES}

The model was implemented in $\mathrm{C}++$ with the capability of interfacing to Gurob 1 or CPLEX 2 solvers with a Visual Basic-supported user interface in Excel. Two heuristic methods were also implemented to provide RTIO with good quality solutions in a shorter time. The rationale behind these heuristics is that the farther back in time a schedule was realised, the less effect it should have in present plans.

1. The optimal method finds the number of trains that maximises profit over the full time horizon, and evidently produces the best solution possible considering all parameters. The optimal problem formulation is made up of around seventy thousand variables and sixty five thousand constraints.

2. Heuristic 1 (H1) solves the problem using a sequence of iterations where train numbers are taken as integers only for the first $D$ periods, and as real numbers for the rest of the time horizon. The solutions from previous iterations are fixed and taken as a starting solution for the next iteration, but the problem is solved for the complete horizon in every iteration. The procedure is repeated until the end of the planning horizon is reached.

1 http://www.gurobi.com/

2 http://www-01.ibm.com/software/integration/optimization/cplex-optimizer/ 
3. Heuristic $2(\mathrm{H} 2)$ uses a sequence of iterations on smaller intervals of $D^{\prime}$ periods. In the first iteration the model is solved for a limited time horizon $\left[0, D^{\prime}\right]$. In the second iteration the model is solved over the horizon $\left[0,2 D^{\prime}-d^{\prime}\right]$ with the solution from the first iteration used to fix the values of $x_{m, p, d, s, t}$ for $t \in\left[0, D^{\prime}-d^{\prime}\right]$. In general, in the $(i+1)$ th iteration the model is solved over the horizon $\left[0, D^{\prime}+i\left(D^{\prime}-d^{\prime}\right)\right]$ with the solution from the $i$ th iteration used to fix the values of $x_{m, p, d, s, t}$ for $t \in\left[0, i\left(D^{\prime}-d^{\prime}\right)\right]$. The process continues until the complete planning horizon is covered.

\section{RESULTS AND DISCUSSION}

The results presented here were obtained using CPLEX 12.1.1 in a 64-bit Intel Xeon CPU with 2 processors of eight cores $(2.27 \mathrm{GHz})$ each and $48 \mathrm{~GB}$ of RAM. The results of the grade preservation algorithm outlined at the end of subsection 2.3 (Figure 1) for 20 iterations show that the grades do converge after seven iterations. The total grade deviation costs are a very small fraction of the total costs (of the order of $10^{-7}$ ) while most of the grade deviation penalties are of around AUD 12.5M per kilotonne. These high penalties and low costs indicate that the deviations from desired grades are very small. Deviation (in \%) from the optimal values is shown in Figure 2(a)

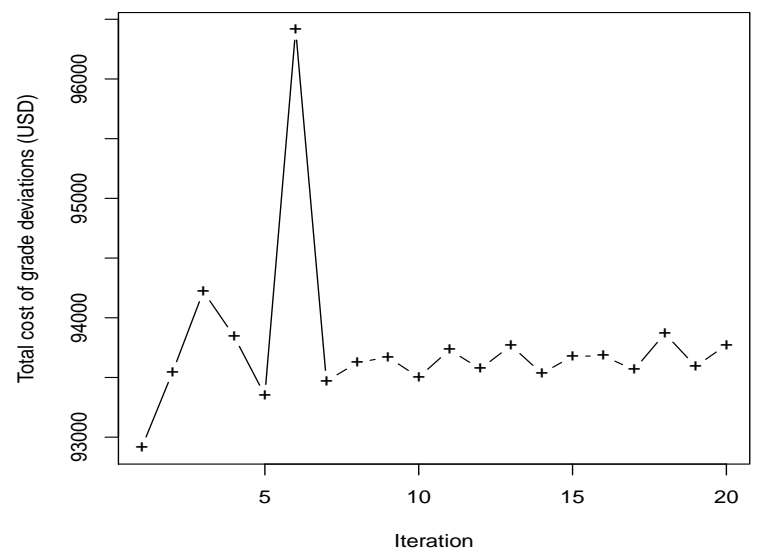

Figure 1: Iron ore grade deviation costs.

H1's quality is worse than H2 in general. As the optimal method was terminated with a gap of $2 \%$, in some cases, the percentage deviation is a small negative number which implies that the heuristics can find better solutions than the optimal method. We find it interesting that even though the heuristics are myopic and do not consider, in the case of H1, that train numbers are always integer, or in the case of $\mathrm{H} 2$, that the full time horizon parameters are disregarded, they manage to find near optimal solutions. Mostly it was H2 that found better solutions than other methods. A comparison of computation times is shown for varying numbers of grade iterations in Figure 2(b). Apart from the obvious increase in time with number of iterations, these results show that $\mathrm{H} 1$ with a high $D$ (25 or 30$)$ and $\mathrm{H} 2$ run faster than the optimal method on the same data set. The speed of $\mathrm{H} 2$ does not vary much as a function of its parameter values, which is to be expected since H2's intervals are smaller and therefore has less variables and constraints. This fact combined with H2's deviation from the optimal value make this heuristic more practical and robust. H1 turns out to be not so reliable.

\section{CONCLUDING REMARKS}

The medium-term rail planning tool presented enables the translation of tactical plans at RTIO into shorter-term, strategic plans by optimising use of various resources like trains, rail tracks and mines. The tool has proved useful in shaping operations, identifying bottlenecks and helping managers understand uncertainties. Grade preservation is an interesting requirement of the 


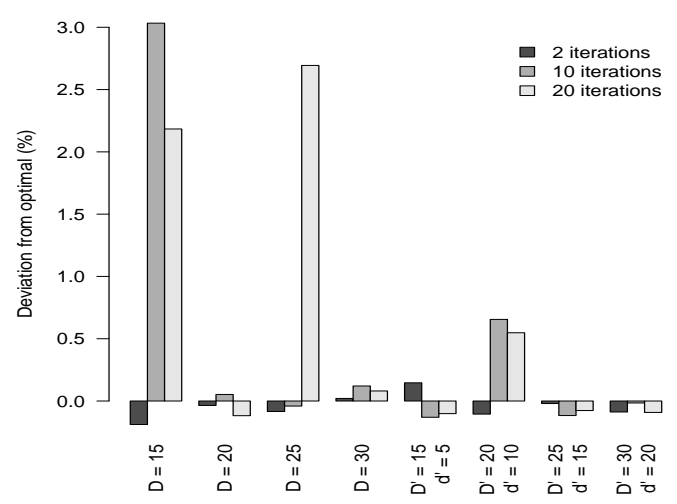

(a) Deviations from the optimal value. Columns marked $D$ refer to $\mathrm{H} 1$, columns marked $D^{\prime}$ and $d^{\prime}$ refer to $\mathrm{H} 2$.

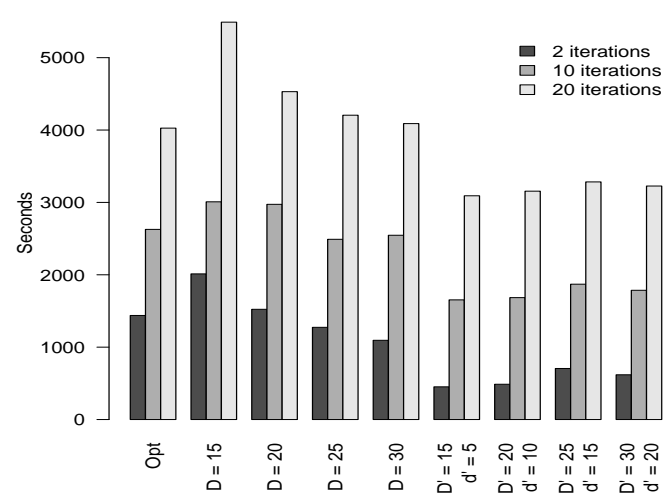

(b) Rail schedule computation times.

Figure 2: Comparison of the three implemented solution approaches.

model, and an algorithm to comply with it was introduced. Results of the optimal implementation and two heuristics were presented, of which $\mathrm{H} 2$ turned out to be more robust and reliable.

Planning time has been cut from five hours to less than one (and sometimes even under 15 minutes) and some enhancements remain in the pipeline. The main of these include shortening the planning horizon to make the tool relevant to day-to-day operations, expressing joint ventures as commitments to commercial partners, and incorporating costing and profit information.

\section{ACKNOWLEDGEMENTS}

We wish to acknowledge contributions from Mr Kerry Munday and Ms Meimei Zhang from Rio Tinto Iron Ore and from Mr. Meng Zhang at various stages of the project.

\section{REFERENCES}

Bilgen, B. and I. Ozkarahan (2007). A mixed-integer linear programming model for bulk grain blending and shipping. International Journal of Production Economics 107(2), 555-571.

Fishman, H. and D. McInnes (2005). Sampling and blending to optimise revenue - a minerals industry perspective. In Proceedings of the Second World Conference on Sampling and Blending 2005, Number 4 in Australasian Institute of Mining and Metallurgy Publication Series, pp. 95-99. Australasian Institute of Mining and Metallurgy.

Li, L. and S. Tayur (2005, February). Medium-term pricing and operations planning in intermodal transportation. Transportation Science 39(1), 73-86.

Rehman, S. and M. Asad (2010). A mixed-integer linear program MILP model for short-range production scheduling of cement quarry operations. Asia-Pacific Journal of Operational Research 27(3), 315-333.

Sandeman, T. and C. Stanford (2010). Integrating optimisation and simulation - a comparison of two case studies in mine planning. In B. Johansson, S. Jain, J. Montoya-Torres, J. Hugan, and E. Yücesan (Eds.), Proceedings of the 2010 Winter Simulation Conference, Winter Simulation Conference Proceedings, pp. 1898-1910.

Ulstein, N., B. Nygreen, and J. Sagli (2007). Tactical planning of offshore petroleum production. European Journal of Operational Research (176), 550-564. 